\title{
La vida de pareja de los adultos jóvenes y las dinámicas socio-demográficas de cambio en el curso de vida. Una revisión teórica para el estudio de sus trayectorias de unión y ruptura
}

\author{
Life in couple of young adults and sociodemographic \\ dynamics of change during the life course. A theoretical \\ revision for the study of their union formation and \\ dissolution trajectories
}

Thaís García-Pereiro • t.garcia.pereiro@unizkm.al

UNIVERSITÀ CATTOLICA «NOSTRA SIGNORA DEL BUON CONSIGLIO»

Recibido: 06/03/2017

Aceptado: 27/05/2017

\section{Resumen}

Recientemente los países occidentales han sido testigos de importantes transformaciones en la transición a la adultez. Los adultos jóvenes están posponiendo los procesos de emancipación y formación de la pareja y la familia. En el caso particular de la vida de pareja, fenómenos como la cohabitación, las parejas sin co-residencia, y la separación o el divorcio, resultan cada vez más diversos y más complejos, pues el calendario, la entrada y salida hacia o desde dichos estados se normaliza y aumenta en frecuencia a medida que avanza el ciclo vital.

Tales niveles de heterogeneidad cuestionan la institucionalización del curso de vida, puesto que las biografías individuales ya no podrían considerarse estables y predecibles. Muy por el contrario, estarían sujetas a variaciones que responden tanto al aumento de la agencia del sujeto como a barreras de tipo estructural, influenciando la construcción de su propia trayectoria vital.

Así, este trabajo el plantear una reflexión teórica desde las perspectivas sociológica y demográfica que pretende servir de base para el análisis empírico de los cambios acaecidos en la vida de pareja de los adultos jóvenes a través de la observación de sus trayectorias de unión y ruptura.

Palabras clave: curso de vida, individuación, trayectorias de unión y ruptura, adultos jóvenes.

\section{Abstract}

Recently, Western countries have witnessed major changes in the transition to adulthood. Young adults are delaying the processes of emancipation and couple and family formation. In the case of couple life, phenomena such as cohabitation, Living Apart Together (LAT), separation and divorce, are becoming more diverse and complex, as the timing, entering and exit to or from such states normalized and its frequency increases with the advancing of the life cycle.

Such levels of heterogeneity challenge the institutionalization of the life course, as individual biographies could not be considered stable and predictable. Quite the contrary, would be subject of variations that respond both to increase of agency as well as structural barriers, both influencing the construction of their own life path.

So, is the main objective of this work to propose a theoretical reflection from the sociological and demographic perspectives as a basis for the empirical analysis of changes in the couple related life of young adults through the observation of their union and breakup trajectories.

Keywords: life course, individualization, union and breakup trajectories, young adults. 


\section{INTRODUCCIÓN}

Durante las últimas décadas, los patrones de formación de uniones han experimentado transformaciones significativas en todas las sociedades occidentales. Algunos de los aspectos que pueden identificarse dentro de este nuevo contexto de emparejamiento son: el retraso en el calendario de entrada a las uniones, la disminución de los matrimonios, el aumento de la cohabitación y de las uniones sin co-residencia (LAT; Living Apart Together), la creciente inestabilidad de las relaciones con el consecuente aumento de las rupturas y del divorcio y de la formación de segundas o posteriores uniones, el incremento de la fecundidad fuera de la institución matrimonial y la creciente importancia de las uniones homosexuales. Esencial para la consolidación de estos nuevos patrones de formación y disolución de la pareja ha sido el protagonismo de los adultos jóvenes como portadores de tales cambios. En este sentido, el papel de las generaciones más jóvenes ha sido de fundamental importancia para la progresiva aceptación y la efectiva configuración de formas alternativas de unión. Los adultos jóvenes apoyan y adoptan con mayor frecuencia modalidades de convivencia diversas del matrimonio, tales como permanecer soltero o cohabitar (Thornton y Young-DeMarco, 2001).

Recientemente, los jóvenes han experimentado cambios concernientes al proceso de transición a la adultez, específicamente en el calendario y el orden de experimentación (secuencia) de los diferentes estados de dicha transición. Los adultos jóvenes han alargado su permanencia en el sistema educativo y han postergado su proceso de emancipación, su entrada a la vida de pareja y la paternidad o maternidad (Elzinga y Liefbroer, 2007; Liefbroer y De Jong Gierveld, 1995). Desde la perspectiva longitudinal o biográfica ha sido demostrado que la postergación de los eventos característicos de la transición a la adultez ha generado un consecuente retraso de las dinámicas de formación de la familia y de la pareja. A pesar de las diferentes explicaciones dadas por la literatura al cambio de las estructuras familiares y las trayectorias de la vida familiar, no existen dudas acerca de la creciente diversidad y complejidad de las trayectorias de unión y ruptura, especialmente en las generaciones más jóvenes.

Lo que caracteriza la contemporaneidad en cuanto a las relaciones es, por una parte, la disminución de la intensidad y el retraso en la formación de la pareja de los adultos jóvenes y, por otro, la formación de las mismas en un contexto más dinámico y complejo que incorpora un amplio abanico de itinerarios y el fenómeno del encadenamiento de parejas. Cuando se menciona la complejidad en los itinerarios, en las trayectorias o en las biografías de unión y ruptura, se hace referencia a la pluralidad de estados y secuencias de tales estados que viven los individuos a lo largo del tiempo. El número y el orden de eventos es muy variable: no compartir residencia y luego decidir vivir juntos; vivir en pareja y posteriormente contraer matrimonio; cohabitar sin llegar a casarse, ni siquiera a largo plazo; separarse en cualquiera de estos momentos e iniciar otra relación completamente distinta a la anterior; éstas entre otras tantas posibilidades. 
Tales niveles de heterogeneidad cuestionan la institucionalización del curso de vida, puesto que las biografías individuales ya no podrían considerarse estables y predecibles. Muy por el contrario, estarían sujetas a variaciones que responden tanto al aumento de la agencia del sujeto como a barreras de tipo estructural, influenciando la construcción de su propia trayectoria vital.

El objetivo principal de este trabajo es plantear una reflexión teórica desde las perspectivas sociológica y demográfica que sirva de base para el análisis empírico de los cambios acaecidos en la vida de pareja de los adultos jóvenes a través de la observación de sus trayectorias de unión y ruptura.

En este artículo se plantea una reflexión teórica rigurosa y sistemática que integra dentro de su marco interpretativo el proceso de individuación de las sociedades occidentales como principal factor explicativo de los cambios demográficos en el ámbito de las relaciones de pareja, reconociéndolas como parte esencial de la Segunda Transición Demográfica, y considerándolas desde una doble visión analítica: el género y el curso de vida. Lo anterior gracias a una revisión integradora que apoya la complementariedad y la pertinencia de una visión más interdisciplinaria a la hora de enfrentarnos a la aprehensión de nuestro objeto de estudio.

El planteamiento anteriormente desarrollado responde, en sí mismo, a diversas finalidades. En primer lugar, retomar el proceso de individuación como explicación tanto de los cambios de las biografías individuales, en general, como de las trayectorias de pareja de los adultos jóvenes, en particular. En segundo, incorporar la perspectiva del curso de vida y de género para interpretar las trayectorias de unión como comportamientos propios de la Segunda Transición Demográfica. Y, finalmente, se pretende ejemplificar conceptualmente la incidencia de los procesos de individualización en las diversas transiciones posibles dentro de las trayectorias de pareja, utilizando el género como intermediario.

El artículo se estructura en cinco partes. En la primera de ellas se recogen los conceptos y principios básicos de la perspectiva del curso de vida, y se introduce la manera en la cual deben ser analizadas las trayectorias de pareja vistas desde esta óptica. En la segunda, se comentan los cambios registrados por la literatura en el curso de vida y el surgimiento del debate referido a la des-estandarización o individuación de comportamientos demográficos. Continuando en la misma línea, la tercera parte retoma la individuación como explicación de las nuevas pautas de formación y disolución de uniones, enfatizando los planteamientos de la STD y los sociólogos de la modernidad tardía. Por último, en la quinta parte se plantea una reflexión teórica que sirve de puente de unión entre las diversas teorías y perspectivas planteadas y cómo de su unificación resulta un marco para la interpretación de las trayectorias de unión y ruptura de los adultos jóvenes. 


\section{REVISIÓN TEÓRICA Y CONCEPTUAL DEL CURSO DE VIDA}

La perspectiva del curso de vida es una orientación teórica que refleja las tensiones existentes entre el individuo y la sociedad y que permite conocer los contextos sociales y temporales de la planeación de la propia biografía, al tiempo que muestra las múltiples formas de adaptación a los cambios tiempo-espacio en las esferas de vida pública y privada (Elder, 1994; Heinz y Krüger, 2001).

No se puede hablar de una teoría unificada del curso de vida (Macmillan, 2005; Elder, 1994). Sin embargo, todos los desarrollos enmarcados dentro de esta perspectiva comparten conceptos claves y principios básicos, imprescindibles para la adecuada comprensión de su objeto de estudio: las biografías.

Se trata de una perspectiva multidisciplinaria, alimentada de las investigaciones realizadas en diversos campos de las ciencias sociales, en especial de la sociología, la demografía, la economía y la antropología. Constituye un área para el estudio de las trayectorias vitales de los individuos desde su nacimiento hasta su muerte.

La metodología del curso de vida se ha alimentado a lo largo del tiempo tanto de desarrollos de tipo cuantitativo como de tipo cualitativo. El primero, gracias a los avances tanto en la recolección como en los instrumentos de análisis de datos longitudinales. Y el segundo, gracias a las aproximaciones biográficas, con el uso y perfeccionamiento de las historias de vida, que permiten obtener una mejor compresión de las diversas maneras por las que los individuos construyen y sobrellevan las experiencias vividas.

Las diversas aportaciones teóricas relacionadas con el curso de vida presentadas a lo largo de este artículo tienen como principal objetivo la introducción de un marco, una perspectiva teórica que puede ser utilizada como guía para el estudio de cualquier tipo de trayectoria biográfica, en general, y de las trayectorias de pareja, en particular.

\subsection{La ordenación y el tiempo, conceptos clave en el curso de vida: trayectorias, transiciones, duraciones y secuencias}

Los elementos temporales de la perspectiva del curso de vida son concebidos a través de la distinción entre las trayectorias, que están conformadas por transiciones; las transiciones, que siguen una determinada secuencia dentro de las trayectorias; $y$, además, a través del calendario y la duración de todas las anteriores.

Las trayectorias y transiciones conforman la base de todo estudio y definen el curso de vida en su totalidad, tanto al largo como al corto plazo. Las trayectorias son dinámicas propias del curso de vida que se extienden a lo largo del tiempo, y tanto su inicio como su final están pautados por transiciones. Estas últimas son más cortas en cuanto a su duración e indican el cambio de un estado o rol a otro. Cada cambio de estado, que marca una tran- 
sición, puede representar un giro en la trayectoria vital, y puede, a su vez, estar influenciado por otras trayectorias o transiciones, o conllevar a posteriores consecuencias en la vida de los individuos.

En palabras de Elder (1994: 6):

"In concept, the life course generally refers to the interdependence of age-graded trajectories, such as work and family careers, that are subject to changing conditions and options in the larger world; and to short term transitions, ranging from birth and school entry to retirement. Each trajectory consists of a series of linked states, as in linked jobs in a work history. A change in state thus marks a transition, a transition to one job to another, for example. Transitions are always embedded in trajectories that give them distinctive meaning».

Las trayectorias vitales están formadas por diversas transiciones con estados conectados unos con otros a lo largo del tiempo, razón por la cual, un registro que permita seguir dichas trayectorias, debe compilar las fechas de entrada y salida de cada estado, su duración, y su vinculación con otras transiciones de trayectorias paralelas.

El concepto de duración responde al de transición y se refiere al tiempo de permanencia en un estado antes de que se produzca el cambio a uno distinto del anterior. Es de esperar que cuanto mayor sea tal tiempo de permanencia en un estado, mayor será la probabilidad de continuidad en el mismo.

Otro aspecto importante a considerar es que los cambios de un estado a otro dentro de las biografías de los individuos siguen un determinado orden al que se le denomina secuencia. La secuencia se refiere al orden en el cual son experimentadas las diversas transiciones a lo largo del curso vida y responde a una lista ordenada que identifica los marcadores de estado a medida que transcurre el tiempo.

Si el objetivo es estudiar la dinámica de pareja de los individuos a lo largo de su curso de vida se debe considerar: 1. Que las trayectorias tanto de unión como de ruptura están conformadas por una serie de transiciones que marcan el paso de un estado a otro; 2. Que los estados son los marcadores relacionados con la vida en pareja: la soltería, la cohabitación, el matrimonio, la separación y el divorcio; 3. Que la secuencia de eventos corresponde al encadenamiento en el tiempo de la diversidad de estados de unión y ruptura antes mencionados.

\subsection{Los cuatro principios básicos del curso de vida: las biografías, su contexto, calendario y vinculación}

\subsubsection{Las vidas dentro de un contexto temporal y espacial}

Las coyunturas de tipo histórico condicionan de diferentes maneras las vidas de los individuos. Los exponen a vivir a ciertas edades determinadas circunstancias que condicionan 
de diferente manera sus vidas, con lo cual terminan viviendo «mundos distintos» de cara al tipo de experiencias vividas, en el sentido en que dichos acontecimientos producen cambios en las oportunidades y opciones de los individuos. De esta manera, los cambios sociales generan diversas consecuencias en las biografías individuales, consecuencias que pueden ser de momento e, incluso, de futuro.

Como se mencionó anteriormente, el curso de vida relaciona la biografía individual con su propio contexto temporal. Esto es posible gracias a la identificación de las formas por medio de las cuales ciertas transformaciones pueden afectar las vidas de los individuos, dichos efectos pueden ser de tres tipos: el efecto cohorte, el efecto período o el efecto edad. Elder (1994: 8) explica las diferencias entre cada uno de estos efectos: «Historical effects on the life course take the form of a cohort effect in which social change differentiates the life patterns of successive cohorts... History also takes the form of a period effect when the effect of change is relatively uniform across successive birth cohorts. A third type of effect occurs through maduration or aging».

No obstante la presencia de cualquiera de los tres efectos del contexto temporal, también es importante considerar las variaciones espaciales o territoriales. Los efectos tendrán consecuencias distintas sobre las biografías en función al lugar en el cual se experimenten. Con lo cual es probable que individuos de la misma cohorte experimenten cambios en sus trayectorias y transiciones de diversa naturaleza si han vivido este momento en espacios territoriales diferentes.

\subsubsection{Las vidas dentro del calendario social y de género}

El significado social de la edad es de particular importancia en la estructuración del curso de vida puesto que influye en el momento, tanto esperado como real, de entrada o salida de los roles sociales y eventos vitales. Su influencia en la estructura de las biografías es visible a través de las normas de edad, las sanciones y presiones sociales, y los internalizados calendarios de ocurrencia de eventos.

El término calendario social engloba el inicio y el final de un estado, así como sus duraciones y secuencias, basándose en unas expectativas construidas socialmente y referidas a la edad, refiriéndose al momento de la vida considerado más adecuado para experimentar ciertos eventos (Elder, 1994). Entonces, podría hablarse de un concepto normativo de calendario social, un calendario de "cumplimiento" de eventos durante el curso de vida que especifica el momento apropiado en el cual los individuos deben educarse, insertarse en el mercado laboral, independizarse, encontrar pareja, casarse, tener hijos, jubilarse, entre otros.

Además de la edad, el género también debe considerarse un moldeador de comportamientos sociales esperados. Existen importantes presiones tanto en el calendario, como en la duración y secuencia de eventos del curso de vida, relacionados con las expectativas so- 
ciales según el género (Widmer y Ritschard, 2009; Heinz y Krüger, 2001). La sociedad ha asignado un patrón de comportamiento deseado y adecuado a hombres y mujeres; patrón que no sólo difiere para cada sexo sino que, además, ha limitado y sancionado tradicionalmente las variaciones u omisiones en el comportamiento, especialmente en el caso de las mujeres y visible en su papel dentro de la configuración familiar.

Investigaciones en la temática (Widmer y Ritschard, 2009; Elzinga y Liefbroer, 2007; Scherger, 2007; Brückner y Mayer, 2005; Fussell, 2005; Liefbroer y De Jong Gierveld, 1995), cuestionan la existencia de un curso de vida normalizado y estandarizado. Argumentan que, desde finales de la década de los sesenta, los arreglos de las biografías individuales han cambiado, convirtiéndose en arreglos cada vez más agenciados por el sujeto, más dinámicos y menos normativos. Tales estudios se preguntan hasta qué punto el curso de vida ha perdido sus marcos determinantes (edad, género) e introducen la existencia de un proceso continuo de negociación propio y compartido, tanto con otros individuos como con las oportunidades y restricciones de su entorno. Las evidencias que sustentan este argumento se basan en la creciente heterogeneidad de patrones y las variaciones de tiempo de ocurrencia, duración y secuencia de diversos eventos observados a lo largo del curso de vida de los individuos.

Heinz y Krüger (2001: 41-42) explican claramente el reciente fenómeno de la pérdida de predictibilidad de la estructura del curso de vida: «The predictable rhythm of life has begun to erode with the extension and change of transition processes between the life spheres of education, family, work and retirement. This means that the standard model of the life course is losing its empirical validity. A less ordered, more flexible life course is in the making on both sides of the Atlantic. Institutionalized markers give way to wider scope for manoeuvre in life course transitions.»

El cuestionamiento de la existencia de cursos de vida estandarizados en las sociedades modernas, se relaciona estrechamente con el siguiente principio a desarrollar: la capacidad del sujeto para tomar las riendas de su propia biografía.

\subsubsection{Agencia del sujeto y toma de decisiones}

A medida que avanzan los procesos de modernización en las sociedades occidentales, los diferentes arreglos del curso de vida se vuelven más dinámicos, menos estandarizados y más dirigidos por el sujeto (Heinz y Krüger, 2001). Y es ante estos sucesos que las investigaciones más recientes del curso de vida se proponen analizar los procesos de negociación, que tienen lugar entre el individuo, las redes sociales, la estructura de oportunidades y las instituciones.

Los individuos viven dentro de un mundo que les ofrece tanto oportunidades como restricciones. Entonces, durante el transcurso de sus vidas, se ven en la necesidad de entablar un diálogo de negociación constante inmersos dentro de un proceso de construcción de su 
biografía que se debate entre las presiones de tipo estructural que reciben del exterior, y sus propios planes y expectativas (Elder, 1994).

Dentro de la perspectiva del curso de vida el concepto de agencia del sujeto constituye un nuevo ángulo de interpretación de las biografías porque dota al sujeto de cierto espacio para la acción, aún bajo las presiones que vienen de la estructura que lo circunda, convirtiendo la agencia y la toma de decisiones en aspectos cruciales para el entendimiento de la relación entre el curso de vida y el cambio social (Heinz y Krüger, 2001). De manera que las macro-estructuras no son las únicas determinantes del devenir de las biografías, los propios individuos contribuyen con sus planes y decisiones a la construcción de las mismas, jugando el papel de agentes más o menos activos.

Según Elder y O’Rand (1995: 457): «Despite the empirical evidence on links between life transitions, the agency of individuals and their life choices ensure some degree of loose coupling between social transitions and states...Loose coupling reflects the agency of individuals even in constrained situations as well as their achievements in rewriting past journeys in the course of aging.»

Como se mencionó en párrafos anteriores, las transformaciones observadas durante el curso de vida (en cuanto a la diversidad del calendario de las trayectorias y la duración y secuencia de sus respectivas transiciones), han sido explicadas por la literatura como consecuencias esperadas de la creciente capacidad de los individuos para dar forma a sus biografías. Este argumento se desarrolla con mayor detalle en el próximo punto del presente artículo, allí se plantean los cambios en la estructura del curso de vida como un nuevo debate dentro de esta perspectiva y se explican los planteamientos que sustentan dichas afirmaciones.

\subsubsection{Vidas vinculadas}

A la hora de estudiar el curso de vida las biografías no pueden considerarse de manera aislada. Así como no pueden obviarse las relaciones (directas e indirectas) con el contexto social, económico, cultural o histórico en el que se desarrollan, tampoco puede dejarse de lado su estrecha vinculación con las redes sociales que conforman su entorno. De allí que otro de los principios centrales de esta perspectiva teórica sea el de las vidas vinculadas.

Las vidas de los individuos se encuentran inmersas en un complejo entramado de relaciones sociales, que van desde vínculos familiares o de amistad, hasta aquéllos de tipo laboral. La interacción constante de los individuos con su red social tiene importantes consecuencias en las biografías, pudiendo incluso modificar el curso de las mismas (Elder, 1994).

Ahora bien, este principio no hace sólo referencia a la vinculación y probables consecuencias que pueden causar otras biografías cuando se interceptan con la propia. También 
considera importantes las vinculaciones entre las diversas trayectorias y transiciones del curso de vida de un mismo individuo. Es decir, puede darse el comienzo de una trayectoria, por ejemplo, la familiar, cuando se ha alcanzado cierto nivel de estabilidad en otra, léase, la laboral. Así, no puede leerse una trayectoria, del tipo que sea, sin tomar en cuenta otras trayectorias o transiciones que podrían actuar sobre ella influenciando directa o indirectamente su devenir.

\section{CAMBIOS EN LA ESTRUCTURA DEL CURSO DE VIDA. NUEVOS DEBATES: ESTANDARIZACIÓN VS. DES-ESTANDARIZACIÓN O INDIVIDUACIÓN}

\subsection{El surgimiento del debate: conceptos claves del cambio ¿bases empíricas?}

Los términos de estandarización, des-estandarización e individuación son claves dentro de los debates actuales del curso de vida. El debate se centra en el cambio que están experimentando las biografías individuales. Estos cambios, según los científicos, se encuentran estrechamente relacionados con los procesos de des-estandarización y la ruptura de secuencias de trayectorias de vida, en otras palabras, se trata del reemplazo de la biografía estándar por la biografía elegida.

El curso de vida está siendo objeto de la des-estandarización tanto en lo referente al orden de sus fases como en términos del carácter lineal de su evolución a lo largo de la vida. En un contexto tal, se asume que algunos individuos podrían dejar de vivir ciertas etapas, o posponerlas, o cambiar rápidamente hacia otras, o regresar a vivir una etapa anterior nuevamente.

Se habla también de que el curso de vida se está convirtiendo en una experiencia cada vez menos colectivizada, pues su des-estandarización lleva aparejado un proceso de flexibilización de las biografías. Una de las perspectivas teóricas que somete a discusión este proceso es la teoría de la individuación. Desde esta perspectiva, tales cambios están siendo reportados con gran énfasis en la literatura. Por lo pronto, se explicarán cada uno de los debates, enfatizando sus definiciones y describiendo las implicaciones que comportan dentro de las biografías individuales.

Dentro de esta temática, los desarrollos anteriores a 1960 se caracterizan por el uso del término estandarización, refiriéndose a la creciente uniformidad en los patrones de las diferentes trayectorias experimentadas durante el curso de vida (Liefbroer y De Jong Gierveld, 1995). Se habla de patrones en los cuales la heterogeneidad es prácticamente inexistente, patrones que imponen regularidades en los eventos y que permiten identificar una serie de transiciones ordenadas en las biografías de los individuos (Widmer y Ritschard, 2009; Fussell, 2005; Modell, Furstenberg y Hershberg, 1976). Para este momento, se considera el 
curso de vida de manera totalmente lineal, es decir, llena de transiciones y trayectorias en las que el calendario de los eventos es poco variable y un evento sigue al otro en un orden predeterminado.

Son diversas las explicaciones dadas a las causas asociadas a la estandarización del curso de vidaํ․ Meyer (1986) la atribuía a la creciente intromisión del Estado en la vida de los individuos y a la también creciente institucionalización de la vida social. Por su parte, Kohli (1986) argumentaba que la mayor predictibilidad de las biografías respondía a la organización de los servicios públicos y a la estructuración por edad del sistema económico-laboral. De manera más general, Buchmann (1989) sugería que la estandarización se debía a los procesos de racionalización de la economía y a los calendarios normativo-institucionales que establecían los lazos entre los diversos mundos vividos escolar, laboral y familiar, entre otros.

Kohli (2007, p.257) sintetiza de manera muy clara lo que para el momento reportaban las investigaciones: "In life course terms, the model consisted in a normal work biography of continuous full-time employment and long job tenure for most of the male population ith most women gravitating around a male breadwinner with various forms of limited engagement in paid work or none at all - and in a normal family biography set in motion by early and almost universal marriage and childbearing».

Así, los elementos que han caracterizado la estandarización del curso de vida han sido tanto la uniformidad, gracias a su carácter fijo en el calendario y en la secuencia de eventos; como la universalidad, dado que los mismos comportamientos han sido identificados en la mayor parte de la población.

La estandarización del curso de vida también ha sido leída en términos de biografías normalizadas (Kohli, 2007), que se traducen en la observada carencia de variaciones tanto en el calendario, como en la ordenación y concatenación de eventos biográficos. Como afirma Scherger (2007: 110): "One characteristic of the temporal structure of societies (of which the life course is one aspect) is the extent to which social processes are standardized, i.e. the extent to which the incidence of transitions, and their beginning, end, duration, succession and rhythm, are fixed.»

Macmillan (2005: 15) resume las implicaciones de la existencia de un curso de vida estandarizado desde el punto de vista teórico y empírico. Así comenta que: «From a theoretical standpoint, it draws our attention to macro-level social change and the role of institutions in the structuring of the life course...Empirically, a standardization thesis suggest homogeneity in both the overall structure of the life course, as well as the implications that it has for individual experience.»

\footnotetext{
${ }^{1}$ Aquí se mencionarán brevemente porque no es el objetivo ahondar en las posibles causas sino más bien retomar debates conceptuales.
} 
A partir de los años sesenta, la idea de un curso de vida estandarizado pierde su fuerza. Los desarrollos posteriores a esta época dejan de lado el término estandarización para referirse a los de des-estandarización o individuación ${ }^{2}$, procesos que describen de manera más adecuada los cambios acaecidos para el momento en las biografías de los individuos (Widmer y Ritschard, 2009; Liefbroer y De Jong Gierveld, 1995; Modell, Furstenberg y Hershberg, 1976).

Los investigadores observan cómo la tradicional secuencia de eventos biográficos de tipo educativo, laboral, familiar y de retiro son sustituidas por cursos de vida más flexibles, menos predecibles, estables y ordenados, y más individuados (Widmer y Ritschard, 2009; Brückner y Mayer, 2005). Esto significa que el curso de vida estandarizado cede el paso a un curso de vida más influenciado por la elección de la propia biografía, en la que el crece el peso de la toma de decisiones relacionadas con el cuándo y el cómo de las transiciones y trayectorias vitales. En este sentido, se podría hablar del abandono de la idea de un curso de vida lineal por uno algo más caótico, complejo y heterogéneo, principalmente de carácter variante.

Basándose en los postulados mencionados en el párrafo anterior, es lógico que la explicación atribuida al concepto de individuación encuentre su centro en la modernización de las sociedades, más específicamente en el ejercicio individual de una mayor libertad concerniente a la toma de las propias decisiones. En este sentido, tanto Giddens (1991) como Beck (1992) comentan cómo el proceso de modernización ha desvinculado a los individuos de los fuertes lazos sociales y culturales que los constreñían a la hora de tomar las riendas de sus vidas. No es que se trate de la completa desaparición de constreñimientos de tipo estructural, más bien se refiere a la pérdida de centralidad de las directrices externas al individuo a favor de la propia toma de decisiones. No se trata, en términos puros, de una total autogestión de la biografía personal, la gestión del proyecto vital se trata de una conjunción entre la parte elegida y la parte producida socio-culturalmente.

Dada la creciente importancia de la agencia del sujeto, las biografías individuales se convierten en proyectos de carácter más deliberado. Aunque los individuos continúan viviendo dentro de un contexto que les condiciona, tales condicionamientos pierden fuerza y se vuelven menos directos y universales, y más difusos. Visto de esta manera, la existencia de un curso de vida individuado lleva a retomar inquietudes científicas, relacionadas con aspectos fundamentales del debate referido a las sociedades contemporáneas.

Ahora bien, es necesario considerar las limitaciones propias al proceso de individuación. La principal radica en que la individuación es una noción parcial, relativa y gradual, en el sentido que, por lo general, no toda una sociedad está completamente individuada, desde el punto de vista de sus comportamientos. Así, ciertos grupos de la población pueden ser

\footnotetext{
${ }^{2}$ Aún cuando suelen utilizarse indistintamente, no son conceptos intercambiables o, al menos, no lo serán para esta investigación. Refieren a marcos causales diversos, y serán explicados y diferenciados más adelante.
} 
los pioneros de la individuación, puesto que las oportunidades para una acción individual vinculada al concepto de agencia del sujeto no se encuentran igualmente distribuidas entre todos los colectivos (Scherger, 2007).

Al contrario de lo observado para la estandarización, la creciente individuación del curso de vida se evidencia en la variabilidad, la heterogeneidad y la pluralidad de las trayectorias y transiciones, y sus respectivas entradas, salidas, duraciones y secuencias (Shanahan, 2000).

La literatura fundamentada en la óptica de la des-estandarización e individuación, no trata de manera exclusiva los términos relativos al cambio de la estructura del curso de vida. Por el contrario, son numerosas las referencias a otros conceptos con ellos asociados, como el caso de la institucionalización/des-institucionalización, pluralización, diferenciación. No parece existir una convención en cuanto a su uso y se puede identificar una falta de precisión cuando se hace referencia a alguno de ellos, utilizándolos indistintamente. Sin embargo, se han identificado en trabajos más recientes importantes esfuerzos de diferenciación conceptual, indispensables para una correcta operacionalización y para la comprobación empírica de la existencia o no de estos fenómenos en las biografías de los individuos (véase, por ejemplo: Brückner y Mayer, 2005; Scherger, 2007).

Interesa dejar claro que la individuación se considera un marco explicativo más amplio que engloba la des-estandarización y los procesos subyacentes de diferenciación, heterogeneidad y pluralidad de las biografías, siendo estos últimos indicadores del cambio. Como se explica a continuación, se considera la individuación como proceso detonante del cambio. Se ha hablado ya de la individuación como teoría que responde a las recientes transformaciones observadas en la estructura del curso de vida, y a continuación se introducen las respuestas que aporta a las transformaciones en la esfera familiar y de pareja.

\section{EL PROCESO DE INDIVIDUACIÓN ASOCIADO A LA FORMACIÓN Y DISOLUCIÓN DE LA PAREJA}

\subsection{Los cambios familiares vistos desde la demografía}

Dentro de la revisión de la literatura científica concerniente al cambio familiar desde una perspectiva demográfica, sistemáticamente se encuentra como referencia obligada la Teoría de la Segunda Transición Demográfica (STD). Salvo la anterior, no se identifican otros intentos recientes por explicar el cambio demográfico familiar en su conjunto, aunque si pequeñas teorías sobre los determinantes de ciertos fenómenos en particular, como el aumento de los divorcios o las cohabitaciones. Con lo cual, la STD se convierte en el único esquema que busca comprender simultáneamente el cambio del conjunto de variables que integran la transformación de la vida familiar y su relación con las transformaciones demográficas. 
El término Segunda Transición Demográfica fue concebido originalmente por Van de Kaa y Lesthaeghe (1987) para dar cuenta del conjunto de transformaciones experimentadas dentro del seno de la familia occidental a partir del 1960. Al finalizar la Segunda Guerra Mundial, y luego de un período de recuperación caracterizado por el auge de la familia, gran parte de los países europeos, y también los Estados Unidos, empezaron a dar signos de importantes modificaciones en ciertos comportamientos demográficos concernientes a las relaciones familiares. Entre ellos se encuentran: por una parte, el aumento del divorcio, el descenso de los matrimonios, la extensión de las cohabitaciones y de los nacimientos fuera de la institución matrimonial y la considerable reducción de la fecundidad; y por otro, el retraso en el calendario, modificaciones observadas gracias al aumento en la edad promedio de inicio de la vida conyugal y de la reproducción.

En palabras de Lesthaeghe (1995), las transiciones familiares se volvieron más frecuentes, más complejas y menos previsibles. La STD implicó un mayor grado de flexibilidad en las relaciones de pareja, cosa que contrastaba con el modelo más o menos predecible de la centralidad del matrimonio y la escasa incidencia del divorcio. El fundamento de las relaciones familiares dentro del marco de la STD se encuentra en el creciente deseo de realización propia y la consiguiente acentuación de la autonomía individual.

Aunque no existe dentro de la teoría de la STD una referencia particular a los cambios en la estructura del curso de vida y su atribución al paso de una biografía estandarizada a otra menos dirigida (des-estandarizada o individuada), resulta evidente el vínculo entre ambas posturas. Así, coinciden tanto temporalmente como en postulados teóricos, ambas identifican las transformaciones en las biografías de los individuos, una de forma más general y la otra de manera más específica, refiriéndose al cambio familiar, y ambas basan las explicaciones del fenómeno en el surgimiento y consolidación de la sociedad post-moderna, dominada por la exaltación de los valores individuales.

No obstante el debate presente en la literatura demográfica (Cliquet 1991; Coleman 2004); en el cual se discute si se trata realmente de una segunda transición o si, simplemente, es la profundización de las grandes transformaciones que dieron lugar a la primera transición demográfica, Lesthaeghe y Van de Kaa (1987) afirman que las motivaciones subyacentes a la STD fueron totalmente diferentes de aquéllas identificadas en la primera. De acuerdo a sus planteamientos, la STD es de carácter individualista, self-oriented, en la que el germen del cambio radica en la realización individual y la calidad de las relaciones entre los integrantes de la pareja. Mientras que la primera, de carácter altruista, child-oriented, implicó el fortalecimiento de los lazos familiares, dentro de los cuales los hijos jugaban un rol esencial.

Si bien en su formulación original los cambios demográficos que caracterizan a la STD fueron vinculados a la expansión de valores individualistas, en general, se ha limitado el uso del término STD a la referencia del conjunto de cambios observados en la familia y en las relaciones de pareja de las sociedades occidentales actuales. Ahora bien, a pesar de esta li- 
mitación de uso, que podría ser discutible, Lesthaeghe y Van de Kaa sostienen que, efectivamente, el nuevo régimen resulta de la modificación de las actitudes frente a la familia. Ambos precursores de la teoría de la STD dan importancia a la dimensión cultural con el objeto de explicar el cambio familiar contemporáneo. Esta dimensión cultural no es más que la emergencia de necesidades post-materiales en los ámbitos de la vida familiar. Visto desde esta óptica, el modelo de familia vigente hasta la década de los sesenta experimenta una re-orientación debida a un cambio ideacional. Se pasó entonces de un sistema sociopolítico regulado por una fuerte estructura normativa, a otro en el que se exalta la libertad de los individuos en la toma de decisiones (Lesthaeghe, 1995, 2004; Van de Kaa 2002, 1987).

Dentro de este nuevo contexto ideacional, y como consecuencia del mismo, la esfera privada experimentó significativas transformaciones. La institución matrimonial comenzó a perder su fuerza como único modelo de establecimiento de compromisos fijos. La relación de pareja, entendida de manera general, pasó a ser escenario de debate y lugar de constante evaluación, basada en las aspiraciones de realización personal de los individuos. En un contexto como éste, los individuos son libres de decidir si forman pareja o no, cuánto tiempo permanecen en ella, si se casan o cohabitan, si tienen hijos y cuándo los tienen; todo lo anterior en función de una evaluación costo-beneficio que obtiene sus cimientos de la idea del desarrollo individual (Van de Kaa, 2002).

Si bien es cierto que en la teoría de la STD el orden cultural del cambio familiar ocupa un lugar preponderante, no se puede negar que también considera que los cambios demográficos no pueden ser entendidos fuera del marco estructural en el que sucedieron. En este sentido, Lesthaeghe y Surkyn (2004) sostienen que los cambios familiares no hubieran sido experimentados sin la existencia de tres revoluciones, también iniciadas a partir de los años sesenta: la revolución contraceptiva, la revolución sexual y la revolución de género. La invención de los métodos anticonceptivos hormonales permitió posponer el inicio de la vida reproductiva hasta el momento deseado, sin necesidad de detener la vida sexual. La revolución sexual, cuestionó el establishment de la exclusiva legitimidad de la vida sexual dentro del matrimonio y la idea de que su única finalidad válida era la procreación. Por su parte, la revolución de género dio pié al cuestionamiento del modelo patriarcal, y su vinculación con la expansión educativa y laboral de las mujeres, constituyó un motor hacia la autonomía de las mismas en la toma de decisiones conyugales y reproductivas.

\subsection{Las transformaciones de las relaciones de pareja según los sociólogos de la modernidad tardía}

Los cambios en la conformación de las parejas y de las estructuras familiares no sólo llamaron la atención de los demógrafos. Otros científicos sociales, específicamente, economistas y sociólogos también intentaron encontrar explicaciones a los cambios acaecidos en la vida familiar. En el campo de la teoría económica, los estudios de familia se nutrieron de los trabajos de Becker (1981) y la New Home Economics, los cuales, a pesar de recibir extensas críticas desde filas feministas, suscitaron gran interés por las relaciones familiares. 
Por su parte, desde la teoría sociológica, dichos cambios fueron estudiados por los que han sido considerados como los más importantes teóricos de la modernidad tardía: Anthony Giddens, Ulrich Beck y Beck-Gernsheim. Estos autores introdujeron las relaciones entre los hombres y mujeres y la vida familiar como componentes del cambio social en sus primeros trabajos de los grandes procesos sociales de finales del siglo xx. Es así como, en los noventa, producen dos obras dedicadas particularmente a la pareja y la familia: La transformación de la intimidad y El normal caos del amor. La publicación de ambos trabajos tuvo dos grandes efectos: influenció en gran medida el desarrollo de la producción científica reciente sobre temáticas familiares, e introdujo los cambios en las relaciones de pareja como dimensión central para una aprehensión más adecuada del cambio social contemporáneo.

A diferencia de otros desarrollos, el principal objetivo de los trabajos tanto de Giddens como de Beck y Beck-Gernsheim no es explicar los cambios de la familia, más bien buscan identificar las causas subyacentes a una mayor individualización en el ámbito de las relaciones interpersonales y en la vida familiar de fines del siglo xx. Si bien es cierto que sus postulados no constituyen explicaciones de las transformaciones familiares, al mismo tiempo son cruciales para comprender la naturaleza de los vínculos modernos entre hombres y mujeres, e ilustran de buena manera su creciente fragilidad.

Estos sociólogos argumentan que la creciente dependencia existente entre las relaciones íntimas y las decisiones personales es un devenir propio de la modernidad tardía, gracias a la defensa y fuerte valoración de la autonomía individual. Como bien argumenta Giddens (1992: 189-190): «....autonomy means the successful realization of the reflexive project of self -the condition of relating to others in an egalitarian way»; y continúa más adelante: «It expresses a prime difference between traditional and present-day marriage and gets to the heart of the democratizing possibilities of the transformation of intimacy. It applies, of course, not just to the initiation of a relationship, but to the reflexivity inherent in its continuance- or its dissolution». El proceso de individuación libera a los individuos, en cierta medida, de los constreñimientos externos, esto constituye un cambio en la concepción del yo y, por ende, en la naturaleza de los vínculos amorosos, que han sido determinantes en la reconfiguración de las relaciones entre hombres y mujeres.

Beck y Beck-Gernsheim expresan la misma idea pero en términos de cambios en las biografías: «La proporción de posibilidades de vida por principio inaccesibles a las decisiones disminuye, y las partes de la biografía abiertas a la decisión y a la autoconstrucción aumentan. La biografía normal se convierte en una biografía elegida...» (1998:14).

A lo largo del siglo xx fueron abriéndose otras posibilidades para unirse en pareja. Hoy existen múltiples opciones de convivencia, lo que no significa necesariamente la superación radical de un modelo de arreglo familiar por otro, sino que, en nuestra época, coexisten diferentes posibilidades tanto de unión en pareja y como de configuración familiar, que abren un amplio margen de opciones a los individuos. 
Para Giddens (1992) el cambio radical producido en la intimidad es posible de identificar en el pasaje del amor romántico al amor confluente. El primero de ellos, está regulado por las normas institucionales del matrimonio; mientras que el segundo, se basa en la constante negociación de las reglas internas del funcionamiento de la pareja. Con esta idea el autor plantea un amor reflexivo que se desarrolla en el marco de lo que él define como "pure relationship», una relación en la que ambas partes entran por iniciativa propia, y que deciden mantener sólo mientras ambos integrantes piensan que la asociación es lo suficientemente satisfactoria. Un tipo de relación como esta es por naturaleza inestable, pues es objeto de evaluación permanente y su sobrevivencia depende de la superación de las probables contradicciones que puedan existir entre los proyectos personales de cada miembro de la pareja. En palabras del autor (1992:58): «A pure relationship refers to a situation where a social relation is entered into for its own sake, for what can be derived by each person from a sustained association with another; and which is continued only in so far as it is thought by both parties to deliver enough satisfactions for each individual to stay within it.»

Para Beck y Beck-Gernsheim (1998) las relaciones de pareja han experimentado las mismas transformaciones relatadas por Giddens. Pero, para ellos, la fragilidad de las parejas modernas reside en el propio carácter de las nuevas relaciones, léase, en la importancia que ha adquirido el amor en la seguridad existencial de los individuos y en la pérdida de vigencia de los referentes de la sociedad tradicional. Su planteamiento responde a una paradoja: mayor individualismo, mayor búsqueda de la auto-realización, mayor necesidad de establecer lazos con el otro. De esta manera, la identidad moderna se construye sobre dos cimientos: el primero, la fragilidad de las relaciones derivada de la creciente importancia del proyecto personal frente al proyecto de pareja; y el segundo, el casi imperativo de encontrar en cualquier momento de la vida una relación satisfactoria. En este punto, Beck y BeckGernsheim introducen los hijos como último elemento a favor de la estabilidad de las relaciones, puesto que representan la garantía de una relación afectiva permanente.

Los autores identifican tres estadios de las relaciones de pareja entre hombres y mujeres. En el primero de ellos, el núcleo familiar era una comunidad económica en la que ninguno de los miembros de la pareja poseía una opción biográfica independiente. En el segundo, el hecho más característico era la represión de la mujer dentro de la vida familiar en aras de la consecución, por parte del hombre, de una biografía más individuada. En el tercero, a partir de la década de los sesenta del pasado siglo, «...comienza claramente una nueva época en la que los dos géneros (aunque en grados diferentes) pueden experimentar los beneficios y las cargas de la vida propia. En esta fase se producen nuevas oportunidades que apuntan hacia una verdadera pareja entre iguales, si bien obviamente surgen también muchos conflictos con la correspondiente confrontación y aislamiento de los géneros.» (Beck y Beck-Gernsheim, 1998:148).

En un trabajo más reciente Beck-Gernsheim (2003) argumenta que la familia tradicional no ha desaparecido totalmente, pero si ha dejado de ser un modelo central y ex- 
clusivo. Introduce el término familia post-familiar para referirse a las nuevas formas de configuración familiar y convivencia: familias reconstituidas, del mismo sexo, monoparentales, cohabitaciones, parejas sin hijos, entre otras. La principal diferencia en las actuales relaciones de pareja según esta autora radica en la importancia que adquiere la propia voluntad y sus consecuentes implicaciones en el seno de las mismas. Por ejemplo, hoy en día el matrimonio no es más que un acto de pura voluntad, quien decide casarse, lo hace con plena consciencia de la existencia de otras alternativas. Además, frente al matrimonio las parejas parecen adoptar estrategias de reducción de riesgo, en las cuales consideran las altas probabilidades de ruptura de la unión y actúan en consecuencia para que en un futuro puedan decidir libremente si finalizar o no su matrimonio.

Si en algo coinciden tanto Giddens como Beck y Beck-Gernsheim es en los factores que han influido en la conformación de este nuevo tipo de relaciones de pareja. Así, explican como el cambio del modelo de pareja no habría sido posible sin la ocurrencia de otros cambios en otras esferas: la aparición de los métodos de anticoncepción, la creciente presencia de la mujer en la esfera educativa y en el mercado de trabajo, y ciertos cambios demográficos como el aumento de la esperanza de vida. De acuerdo con Giddens, la expansión del uso de métodos anticonceptivos eficientes dio paso al surgimiento de la sexualidad plástica, liberada totalmente de las exigencias reproductivas. Se trata de un tipo de sexualidad que reivindica el placer sexual de la mujer y desvincula al sexo del matrimonio. Siguiendo la línea argumentativa de este autor, la presencia femenina en el sistema educativo y laboral, en conjunción con los movimientos feministas, permitieron que el matrimonio perdiera relevancia como fuente de auto-sustento. Entonces, las mujeres consolidaron su papel como agentes de negociación en la vida conyugal y su nueva independencia económica les concedió las herramientas necesarias para terminar con matrimonios insatisfactorios y para tener la opción, si así lo deseaban, de mantener un hogar con sus propios recursos, dos efectos fundamentales para el surgimiento de la relación pura. El último de los cambios, el aumento de la esperanza de vida, posibilitó retrasos de calendario nunca antes observados en las vidas adultas, como el retraso de la edad a la primera unión y a la concepción del primer hijo.

\section{UNIENDO TEORÍAS Y PERSPECTIVAS: UN MARCO PARA INTERPRETAR LAS TRAYECTORIAS DE PAREJA DE LOS ADULTOS JÓVENES}

\subsection{El proceso de individuación y la Segunda Transición Demográfica vistos desde la perspectiva del curso de vida y las relaciones de género}

Como se ha mencionado en apartados anteriores, el marco de interpretación de la Segunda Transición Demográfica necesita tanto de los procesos de individuación como de 
las relaciones de género para basar en ellos sus explicaciones. El proceso de individuación, especialmente entendido desde la perspectiva del curso de vida, está estrechamente vinculado con los planteamientos de la STD, en tanto que entablan una relación causal. En los indicadores demográficos se reflejan no sólo los nuevos comportamientos familiares y de pareja, igualmente considerados como cambios en la estructura del curso de vida, si no también los procesos subyacentes que generan dichos comportamientos. El surgimiento del individuo como sujeto autónomo, que responde a las nociones de voluntad, elección y libertad personal, constituye el fundamento del proceso de individuación en las sociedades modernas. Cada miembro de la pareja, dentro de su propia dinámica, puede ser visto como un proyecto de vida en curso y, como tal, es sujeto de gestión su propia biografía.

Profundizando en este aspecto, y respondiendo a la parte más ideacional de la teoría de la STD, Bernhardt (2004) argumenta a favor de la importancia de enfatizar los valores como elementos del cambio a nivel micro. Dentro de la Teoría de la STD, la autonomía individual es, en gran parte, la explicación de los cambios observados en los comportamientos demográficos. Sin embargo, la autora también llama la atención acerca de la necesaria inclusión de la perspectiva de género en un marco como la STD, pues ni la autonomía y realización personal, ni la transición de un modelo de familia a otro, ni los cambios recientes en la estructura del curso de vida, pueden ser entendidos sin considerar que tanto unos aspectos como otros son distintos si se observan, por separado, los efectos que han tenido en las vidas de las mujeres o en las de los hombres. Los conceptos detrás del proceso de individuación no son neutrales en lo que al género respecta, tienen distintas implicaciones según sea el caso. Las biografías masculina y femenina devienen de construcciones diversas y, seguramente, también son distintos los significados otorgados por los protagonistas tanto a las experiencias vividas como a las expectativas de futuro.

Ahora bien, retomando de manera específica el caso de las relaciones de pareja, Bernhardt afirma que el fenómeno que se encuentra en el centro de la STD es la fragilidad de las relaciones entre hombres y mujeres, argumento también defendido por los sociólogos de la modernidad tardía. Una fragilidad que deviene del propio proceso de individuación y que, por supuesto, no afecta de igual manera a hombres y mujeres. Existen diferencias considerables entre los proyectos de vida masculinos y femeninos y, la conjunción de dichos proyectos dentro de una unión de pareja, no se encuentra exenta de conflictos.

Las transformaciones de la biografía familiar y de pareja han sido acompañadas por el aumento de la participación femenina en el mercado laboral. De hecho, algunos cambios como la disminución de la intensidad del matrimonio y de la fecundidad y el aumento de las rupturas han sido estrechamente relacionados con el empleo femenino. Sin embargo, Goldscheider et al. (2015) sostienen que estos vínculos están perdiendo fuerza gracias a la revolución de género: a medida que la participación en el mercado de trabajo se convierte en parte natural del curso de vida femenino, las relaciones de género cambian y los vínculos entre los eventos de la biografía laboral y familiar mutan. 
Según los autores, la entrada de las mujeres al mercado de trabajo genera stress en la vida familiar y de pareja sólo en la primera parte de la revolución de género. La consecuente dificultad de conciliación entre vida laboral y familiar induce a una renegociación del rol de cuidado de la mujer en la familia que puede ser la fuente del retraso de la formación de la pareja, la postergación de la maternidad y el aumento de las rupturas de unión. En la segunda fase de la revolución sucede exactamente el contrario, la mayor participación de los hombres en el ámbito doméstico (tareas del hogar y cuidado de los hijos) reduce la dificultad de conciliación y elimina los obstáculos favoreciendo la formación de la familia. Esta fase de la revolución es aún incipiente en gran parte de las sociedades occidentales, pero muestra señales de progreso en los países nórdicos.

Siguiendo esta línea, Esping-Andersen y Billari (2015) conjeturan que el aumento de la soltería y de la separación y la disminución de la fecundidad son tendencias destinadas a retroceder con el aumento de la igualdad de género. Así, una división equitativa del trabajo doméstico entre hombres y mujeres resulta claramente favorable a la compatibilidad entre empleo y familia.

La aplicación de la perspectiva del curso de vida a las trayectorias de unión y ruptura de los adultos jóvenes necesita que se reconozcan la indivisibilidad de la biografía laboral y de pareja (familia), especialmente en el caso de las mujeres, y los efectos que los cambios en los roles de género pueden causar en los comportamientos.

En otros tiempos, el matrimonio constituía sinónimo de certeza, una certeza basada en una alianza que duraría para siempre, muy a pesar de las valoraciones positivas o negativas que de él pudieran surgir. El matrimonio implicaba duración e hijos, por lo que no se admitía la separación. Por el contrario, en la actualidad, los procesos tanto de formación como de disolución de uniones han sido permeados por la libertad de elección, por la individuación. Como se ha mencionado anteriormente, una unión basada en semejante formato lleva implícita la posibilidad de disolución, pues el descontento y la falta de satisfacción son ahora motivos para acabar una relación.

En este contexto, ya no tiene sentido hablar de una trayectoria familiar fija, en la que los jóvenes salen de casa de sus padres, se casan y tienen hijos, todo dentro de un calendario más o menos regular. Menos sentido tiene aún si se trata de hilar más filo, como es el caso de este artículo, profundizando sólo en las trayectorias de pareja. En ellas sería muy probable encontrar continuas entradas y salidas de relaciones de distintos tipos, LAT's, cohabitaciones, matrimonios e, incluso, uniones post-matrimoniales. Es así como el objetivo principal del siguiente sub-apartado es el de reconfigurar el estudio de las trayectorias de unión y ruptura de los adultos jóvenes, las generaciones que mejor reflejan el cambio en las relaciones de pareja, a través del marco de la individuación y ubicándolas dentro de la perspectiva del género y el curso de vida. 


\section{A MODO DE CONCLUSIÓN: UNA CONTEXTUALIZACIÓN TEÓRICA PARA LAS TRAYECTORIAS DE PAREJA DE LOS ADULTOS JÓVENES}

El presente artículo no pretende construir nuevas aportaciones teóricas o delinear una propuesta empírica novedosa, objetivos que se retienen extremamente ambiciosos considerando la vasta complejidad de la temática. Hasta este punto, el objetivo ha sido dotar al estudio de las trayectorias de unión de un marco conceptual e interpretativo adecuado y riguroso. En este apartado se busca explicar cómo los diversos planteamientos teóricos aquí descritos pueden ser leídos en clave de conjunto, incorporándolos dentro de un marco de análisis para las trayectorias de unión de los adultos jóvenes. Aquí reside el interés de este apartado, que más que una recopilación de aportes dentro de la literatura científica, pretende servir de unión entre una teoría multidisciplinaria (la individuación), una demográfica (STD), dos ejes de análisis (género y curso de vida) y las dinámicas biográficas particulares. Es decir, pretende responder a cómo los planteamientos teóricos en el área del curso de vida y la formación y disolución de la pareja, y los ejes biográfico y de género, pueden contribuir a la interpretación de las trayectorias de unión y ruptura de los adultos jóvenes al servicio de posteriores estudios empíricos.

Se ha intentado desplegar el argumento principal de lo general a lo específico, como resultado natural de la reflexión posterior a la revisión bibliográfica realizada. Se ha intentando resaltar la complementariedad de los postulados de la teoría del curso de vida, el debate de individuación surgido en su seno, y las teorías del cambio familiar, y, se comentará aquí, cómo esta especie de unificación sirve para explicar las trayectorias de unión de los adultos jóvenes. El producto es la estructuración de un discurso que deviene de los desarrollos teóricos recogidos, vinculándolos lógicamente, cuando, por lo general, son tratados de manera separada.

Los nuevos modelos familiares se caracterizan por la pluralidad de formas de convivencia y por la diversidad de itinerarios para formar y disolver uniones. Cada vez es menos frecuente el curso de vida que comenzaba con la emancipación de los jóvenes vía matrimonio y seguía con el nacimiento y crianza de los mismos siempre dentro de dicha institución. Hoy en día los itinerarios son más heterogéneos, múltiples y, más importante aún, reversibles.

Dentro de un contexto en el cual cada uno es más o menos responsable de la construcción de su propia biografía, su planificación conduce a una amplia diversidad de itinerarios posibles y, por ende, a una flexibilización de los modelos.

La población en la que los cambios son más evidentes es la adulta-joven. Los adultos jóvenes desempeñan un importante papel dentro de las transformaciones en la dimensión de la pareja. Son generaciones que han vivido décadas de grandes cambios y, como consecuencia, se han convertido en los portadores de los nuevos valores de individuación y en 
los mejores sujetos para estudiar el comportamiento familiar y de pareja. Recientemente, los jóvenes han experimentado cambios concernientes al proceso de transición a la adultez, han alargado su permanencia en el sistema educativo y han postergado su emancipación, su entrada en la vida de pareja y las nuevas paternidades o maternidades. No existen dudas acerca de la creciente diversidad y complejidad de las trayectorias de unión y ruptura en las generaciones más jóvenes.

La teoría de individuación del curso de vida, en general, y de las relaciones de pareja, en particular, planteadas en esta reflexión teórica, no deben leerse en términos de la mayor libertad de decisión de los actores en las sociedades contemporáneas. Más bien, deben interpretarse como los resultados observables de dichas decisiones. Es decir, a efectos de interpretación empírica, la individuación existirá únicamente si en estudios posteriores se verifica que existe, dentro de las trayectorias de unión y ruptura de los adultos jóvenes, una mayor variación, pluralidad y heterogeneidad al observar el calendario de las diversas transiciones y sus respectivas secuencias.

Con base en todos los argumentos explicados hasta el momento, el estudio de las trayectorias de unión y ruptura debe comprender el conjunto de las diversas transiciones (de entrada y salida de eventos) relacionadas con la formación y disolución de uniones. Específicamente, interesan las transiciones de este tipo vividas en la etapa adulto-joven, en la cual los acontecimientos propios de la vida de pareja concentran su mayor actividad. Siendo la finalidad central el identificar, dentro de esta etapa de la trayectoria vital, las entradas y salidas de eventos de pareja: formación de LAT's, cohabitaciones, matrimonios, y disolución de las mismas. Pero no solamente interesa la identificación aislada de cada tipo de unión junto a su incidencia, calendario y duración; también se trata de reconstruir y situar estos eventos a lo largo de una línea de vida en unión, para estudiar su secuencia y, de esta manera, determinar las diversas ordenaciones dentro del amplio abanico de concatenación de posibles eventos dentro de las trayectorias de unión.

En las últimas décadas hablar de adultos jóvenes implica hablar de incerteza y de profundos cambios en las transiciones y trayectorias, es decir, en sus biografías. Si bien es cierto que la etapa joven se ha prolongado durante el curso de vida, dicha postergación no ha hecho que aumentar la complejidad, la fragmentación y la de-estandarización de la transición a la adultez. Como consecuencia, la investigación sociológica y demográfica necesita reflexionar profundamente acerca de las características típicas de las transiciones: diversidad (entre individuos y en cada biografía), reversibilidad e inter-conexión.

En el caso de la biografía familiar, las trayectorias de unión y ruptura se conforman a través de la comunión de distintas transiciones, un amplio abanico de posibilidades que va desde la más simple (solteria-1er_matrimonio) hasta otras más complejas (solteria-1era_ cohabitación-ruptura-2da_cohabitación-1er_matrimonio-divorcio-1er_LAT).En estos ejemplos resulta evidente que la mayor heterogeneidad de las trayectorias depende especialmente del carácter reversible de las transiciones que, en el caso de la vida de pareja, 
encuentra su máximo exponente en la creciente y reciente inestabilidad de las relaciones románticas. Sin embargo, es fundamental tener en cuenta que dichas transiciones pueden variar considerablemente en función de su calendario, secuencia de ocurrencia y duración a lo largo del curso de vida, todas ellas fuente de importantes diferencias en las biografías de los adultos jóvenes.

Las transiciones de unión y ruptura no pueden ser estudiadas de forma aislada. Al igual que otros eventos del curso de vida, la formación y disolución de la pareja se relacionan directa e indirectamente con otras trayectorias y transiciones. Dos de los ejemplos más relevantes de la interconexión de las trayectorias biográficas en el ámbito familiar son las interdependencias entre la trayectoria laboral y la formación de la unión, especialmente del matrimonio, y la trayectoria de fecundidad, la transición al matrimonio y su posterior disolución.

Además, resulta indispensable reconsiderar que tanto las biografías, en general, como las trayectorias y transiciones, en particular, responden a un juego de equilibrio entre los propios deseos y decisiones y las constricciones que el ambiente social, económico y cultural ejerce sobre las mismas. De allí que resulte indispensable evaluar el peso de cada una de estas constricciones en el desarrollo del curso de vida.

En una época como la presente, caracterizada por una gran inestabilidad económica, los adultos jóvenes experimentan un periodo de adaptación al entorno en el que alteran sus expectativas y escalas de tiempo para comenzar una vida autónoma. Los cambios en el comportamiento asociado a la formación de la familia, no se han producido al margen de los cambios del mercado laboral, de las novedades en ámbito legislativo e, incluso, de la dinámica del mercado inmobiliario. De aquí la importancia de considerar conjuntamente el desarrollo de nuevos valores (cambio ideacional), las limitaciones de tipo estructural y los cambios en las relaciones de género para explicar las decisiones vinculadas a la vida familiar y de pareja de los adultos jóvenes.

\section{REFERENCIAS BIBLIOGRÁFICAS}

Beck, U. (1992). Risk Society: Towards a new modernity. London: Sage.

Beck, U. y Beck-Gernsheim, E. (1998). El normal caos del amor. Barcelona: El Roure Editorial.

Becker, G. (1981). A treatise on the family. London: Harvard University Press.

Beck-Gernsheim, E. (2003). La reinvención de la familia. En busca de nuevas formas de convivencia. Barcelona: Paidós.

Bernhardt, E. (2004). Is the Second Demografphic Transition a useful concept for dempography? Vienna Yearbook of Population Research, pp. 25-28. 
Brückner, H. y Mayer, K.U. (2005). De-standardization of the life course: What it might mean? And if it means anything, whether it actually took place?. Advances in Life Course Research, 9, pp. 27-53.

Buchmann, M. (1989). The Script of Life in Modern Society: Entry into Adulthood in a Changing World. Chicago: Univ. Chicago Press.

Cliquet, R. (1991). The Second Demographic Transition: fact or fiction?. Strasbourg.

Coleman, D. (2004). Why we don't have to believe without doubting in the "Second Demographic Transition» - some agnostic comments. Vienna Yearbook of Population Research, pp. 11-24.

Elder Jr, G.H. (1994). Time, human agency, and social change: Perspectives on the life course. Social psychology quarterly, 57, 1, pp. 4-15.

Elder, G. y O’Rand, A. (1995). Adult lives in a changing society. Sociological perspectives on social psychology, pp. 452-475.

Elzinga, C.H. y Liefbroer, A.C. (2007). De-standardization of family-life trajectories of young adults: A cross-national comparison using sequence analysis. European Journal of Population, 23, pp. 225-250.

Esping-Andersen, G., y Billari, F.C. (2015). Re-theorizing Family Demographics. Population and Development Review, 41, 1, pp. 1-31.

Fussell, E. (2005). Structuring the transition to adulthood: An entropy analysis of the early life course in the United States, 1880 to 2000. Advances in Life Course Research, 9, pp. 91-122.

Giddens, A. (1991). Modernity and self-identity: Self and society in the late modern age. Stanford: Stanford University Press.

Giddens, A. (1992). The transformation of intimacy: Sexuality, love, and eroticism in modern societies. Standford: Standford University Press.

Goldscheider, F., Bernhardt, E., y Lappegård, T. (2015). The gender revolution: A framework for understanding changing family and demographic behavior. Population and Development Review, 41, 2, pp. 207-239.

Heinz, W. y Krüger, H. (2001). Life course: innovations and challenges for social research. Current Sociology, 42, 2, pp. 29-45.

Kohli, M. (1986). The world we forgot: A historical review of the life course. Beverley Hills: Sage.

Kohli, M. (2007). The Institutionalized Life Course: Looking Back and Looking Ahead. Research in Human Development, 4, 3-4, pp. 253-271. 
Lesthaeghe, R. (1995). The Second Demographic Transition in Western European Countries: an interpretation. En K. Oppenheim Mason y A.-M. Jensen (Eds.), Gender and family change in industrialized countries (18-48), Oxford: Oxford University Press.

Lesthaeghe, R. (2004). Meaning and choice: value orientations and life course decision. Population, 5, pp. 805-806.

Lesthaeghe, R. y Surkyn, J. (2004). When History Moves on: The Foundation and Diffusion of a Second Demographic Transition in Western Countries: An interpretation. 12th Biennial Conference of the Australian Population Association.

Liefbroer, A.C. y De Jong Gierveld, J. (1995). Standardization and individualization: The transition from youth to adulthood among cohorts born between 1903 and 1965. En V. d. B.J.C. y D.F. Dordrecht (Eds), Population and family in the low countries 1994 (57-80), Kluwer Academic Publishers.

Macmillan, R. (2005). The structure of the life course: classic issues and current controversies. Advances in Life Course Research, 9, pp. 3-24.

Mayer, K. (2009). New directions in life course research. Annual Review of Sociology, 35, pp. 413-433.

Meyer, J. (1986). The self and the life course: Institutionalization and its effects. En A. Sorenson, F. Weinert y L. Sherrod (Eds.), Human development and the life course: Multidisciplinary perspectives (199-216), Hillsdale: Lawrence Erlbaum Associates.

Modell, J., Furstenberg Jr, F.F., y Hershberg, T. (1976). Social change and transitions to adulthood in historical perspective. Journal of Family History, 1, 1, pp. 7-32.

Scherger, S. (2007). Destandardization, Differentiation, Individualization? Changing West German Life Courses. Wiesbaden: Verlag für Sozialwissenschaften.

Shanahan, M.J. (2000). Pathways to adulthood in changing societies: Variability and mechanisms in life course perspective. Annual Review of Sociology, 26, pp. 667-692.

Thornton, A., y Young DeMarco, L. (2001). Four decades of trends in attitudes toward family issues in the United States: The 1960s through the 1990s. Journal of marriage and family, 63, 4, pp. 1009-1037.

Van de Kaa, D.J. (1987). Europe’s Second Demographic Transition. Population Bulletin, 42, 1, pp. 3-59.

Van de Kaa, D.J. (2002). The idea of a Second Demographic Transition in Industrialized Countries. Sixth Welfare Policy Seminar at the National Institute of Population and Social Security, pp. 1-32.

Widmer, E. y Ritschard, G. (2009). The De-Standardization of the Life Course: Are Men and Women Equal? Advances in Life Course Research, 14, 1-2, pp. 29-39. 